\title{
The Sonification Space: a Reference System for Sonification Tasks
}

\author{
Luca A. Ludovico, Giorgio Presti \\ Laboratorio di Informatica Musicale \\ Dipartimento di Informatica \\ Università degli Studi di Milano \\ Via Comelico, 39 - 20151 Milano, Italy \\ \{luca.ludovico,giorgio.presti\}@unimi.it
}

\begin{abstract}
Sonification is a fairly new term to scientists who are unaware of its multiple use cases. Even if some general definitions of the concept of sonification are commonly accepted, heterogeneous techniques - significantly different as it regards approaches, means and goals - are available. In this work we propose a reference system useful to interpret already-existing sonification instances and to plan new sonification tasks. This work aims to present a reference system for sonification using the inherent properties in the sonic output rather than the data itself. Validation has been conducted by automatically analyzing available experiments and examples, and placing them on the proposed sonification space, according to time-granularity and abstraction-level dimensions. This work can constitute the starting point for future research on computer-assisted sonification. It will be beneficial to a wide range of readers, in particular those from different disciplines looking at new ways to present and analyze data.
\end{abstract}

Keywords:

sonification, audio, sound parameters, data visualization

\section{Introduction}

Sonification is the transformation of data relations into perceived relations in an acoustic signal for the purposes of facilitating communication or interpretation [1]. In other words, sonification provides a way to represent 
data as sound, namely to convey meaning from a dataset to a listener via sonic interfaces.

The concept of sonification has been widely explored in scientific literature, and most scientists, experts and artists who have been involved with sonification tend to agree on some commonly accepted definitions. For example, in [1] sonification is described as "the use of non-speech audio to convey information", a locution broad enough to embrace different approaches. For example, heterogeneous sonification techniques such as audification, earcons, auditory icons, parameter mapping and model-based sonification are covered by such a definition, since they all represent information/data by using sound in an organized and structured way.

However, our research does not aim at finding a comprehensive description able to embrace different types of sonification, on the contrary we need to identify a number of distinguishing features in order to characterize a number of possible meanings of sonification.

In this sense, the first step is understanding if the instance of a given sonic interface can be considered sonification or not. Sonification has to satisfy the criteria expressed in [2]:

- The sound has to reflect properties and/or relations in the input data;

- A precise definition of how interactions and data cause the sound to change must be provided. Random elements may be allowed, provided that their use is declared and defined;

- Sonification has to be reproducible, so that the same data and interactions/triggers must result in structurally identical sound, which does not imply sample-based identity;

- The system can intentionally be used with different data, as well as in repetition with the same data.

Using this criteria some forms of sonic performance cannot be considered as sonification. For example, real-world acoustics cannot be defined as sonification since there is no external input data; similarly, playing a musical instrument is not a sonification of the performer's emotional state, since it can not be repeated with the identical data, even if the resulting sounds can be seen as a sonification of the interactions with the instrument [2]. 
After defining what sonification is and what is not, we try to identify the goals of the sonification. The aim is not creating a goal-based reference taxonomy, but discovering the needs that sonification could satisfy. An analytical and unambiguous discussion of the subject is not trivial, as goals are often correlated with the means adopted to achieve them. In general terms, we can recognize:

- Artistic purposes, which imply non-trivial aesthetics matters, as discussed in [3]. For example, in modern electronic music the concepts of music and sound are often merged, and musicians have been using data for compositional purposes for a long time, so it can be difficult to distinguish between artistic expression and sonification. In addition, this kind of sonification allows authors to train an aesthetic sense, useful to make the listening experience a more compelling task for the user;

- Sonic plot, defined also as parameter-mapping (and sometimes as modelbased sonification), where data features are accurately mapped to acoustic features (e.g. pitch, inharmonicity, etc.) of sound events or streams. This approach is adopted when precise data tracking is required $[4,5]$, and it is probably the most challenging one due to user-perception issues [6] and signal features [7];

- General system-state description, where no precise discrimination is required, but only main holistic aspects have to emerge [8, 9, 10]. This research field is relatively recent, but is promising especially in the context of affective interaction [11];

- Interactive dataset exploration, namely a kind of data sonification which takes place only when users interact with such data [10]. Possible application fields are complex dataset navigation, improvement of traditional interfaces, augmented reality and assistive technologies [12].

In the next section we will propose a diagram representing a conceptual framework - called the sonification space - where different approaches to sonification can be suitably represented.

\section{The Sonification Space}

Now we introduce the sonification space, namely a graphical representation aiming to highlight goals and approaches of different sonifications 


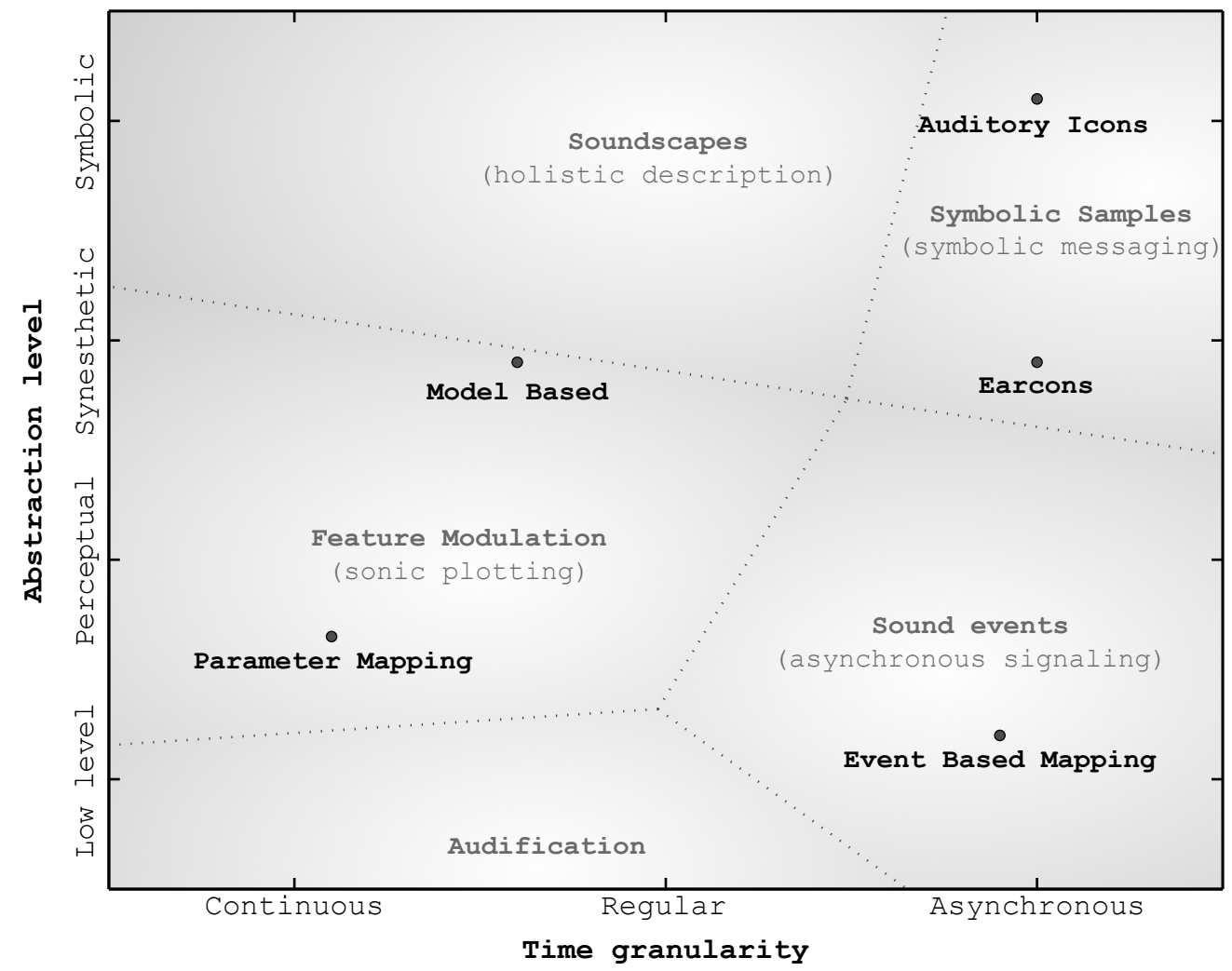

Figure 1: The proposed sonification space, with the identification of areas (shades of gray) and the positioning of some traditional sonification approaches (black circles).

through their sonic outcomes. The idea is proposing such a graphical tool to help the end user understand the sonification model in use and focus on the key audio features. This graph was inspired by [13] and [14] and built starting from the empirical evidences coming from a number of sonification cases. The resulting sonification space is shown in Figure 1.

The $x$ axis represents sound granularity, whereas the $y$ axis is related to the level of abstraction of the sound output. These concepts will be discussed in detail below. By now, in order to give a broad picture of the subject, it is possible to place classical approaches and techniques over the sonification space. The upper area, labelled as Soundscapes, refers to a more abstract and holistic description level, namely the aforementioned general system-state description. The middle area, marked as Feature Modulation 
and Sound events, refers to sonic plot sonification. The right part of the diagram, containing interactive dataset exploration, is labeled as Symbolic samples and Sound events. Finally, Audification can be considered as a direct listening of a dataset, and sometimes this category is not classified as sonification; its vertical position in the diagram is quite arbitrary, since its abstraction level depends on data.

Defining such a space can be helpful in the authoring of a sonification task. In fact, the information to convey, as it regards its level of abstraction and detail, broadly identifies an area over the diagram, which in turn will suggest the best sonification strategies. The former aspect is the core of this paper, whereas the latter requires further investigation.

Even if we can identify the average position of a number of classes over the diagram, it is worth underlining that:

- The sonification space should be considered as a continuum, since no boundaries exist among areas;

- The different sonification techniques mentioned above (represented by black circles in Figure 1) have been placed according to their typical auditory outcome and should be considered as mere examples.

Now a more detailed discussion about the diagram interpretation is called for. In the lower left part of the diagram we find techniques able to convey precise information, provided that proper mappings are chosen. For example, even if most listeners are not able to recognize the exact frequency of a tone, thanks to basic training they can distinguish among main music intervals and with no training at all they can perceive changes in timbre. Moreover, recent literature and current research $[15,16]$ link low-level sound features to emotional responses, thus enabling scenarios of affective interaction.

Unfortunately, these techniques show their limits when mapped features lack in orthogonality $[6,7]$. Besides, as parameter-mapping techniques are often focused on sound synthesis parameters rather than on perceived features, generally they do not exploit the natural ability we developed to gather information from given sound features. For instance, the Doppler effect is instinctively used to evaluate the position of a vehicle approaching, passing by, and receding from the observer. Moreover, sensory dissonance is suggestive of danger in natural environments, because it occurs in the threat and warning calls of many species of animals [17]. Many other examples have been collected in [16]. 
The upper part of the diagram is populated with highly symbolic sounds, suitable to convey general systems-state or multidimensional data. Their applicability depends on an effective use of sound to gather holistic information about the system [8, 9]. For example, a mix of layered soundscapes can trigger somatic markers even if listeners are not aware of sound details $[16,18,19]$. On the other hand, these techniques are not able to provide precise indications about low-level parameters. Moreover, many symbolic aspects of sound are culture-dependent, so it is difficult to create a sonification having a universally accepted meaning.

Finally, a sonification instance may rely on different techniques, spanning all over the identified plane. Different aspects of sonification can be mapped over different areas: a network of vertexes called data bindings can be used to pin the position of used techniques, while a single main feature pins out how the overall outcome should sound or how it should be interpreted. Data bindings can be seen also as the mathematical functions chosen by the sonfication author to translate single data streams into a sound feature.

In this context, the different concepts of data bindings and main features may help distinguish among sonifications which are similar in the used technique but very different in their outcome. For instance, let us consider "Sonifying specific data points in a sequence along with the entire process" by Jonathan Berger and "Iraq Body Count", a sonification by Guillaume Potard of the data coming from a website dedicated to Iraq War, both cited in [20]: in the first case a rising pitch conveys a general idea of the system trend while iconic sounds mark the occurrence of relevant values, while in the second case pitch is controlled by oil price and war deaths trigger sound icons. A representation of both examples can be rendered in the proposed sonification space as shown in Figure 2 (the former example is labeled as "Sonification 17", the latter as "Iraq Body Count").

In both the mentioned cases different levels of abstraction coexist with continuous and discrete features; our plot can help distinguishing the kind of interaction between them. In fact, even if the data bindings of "Iraq Body Count" are located very close to the ones of "Sonification 17", the position of the main features highlights the different goals and outcomes: "Sonification 17 " has a more precise description of the mapped parameter and sounds more like a sonic plot, while the work of Guillaume Potard provides an overview of the war, sounding as an artificial soundscape. 

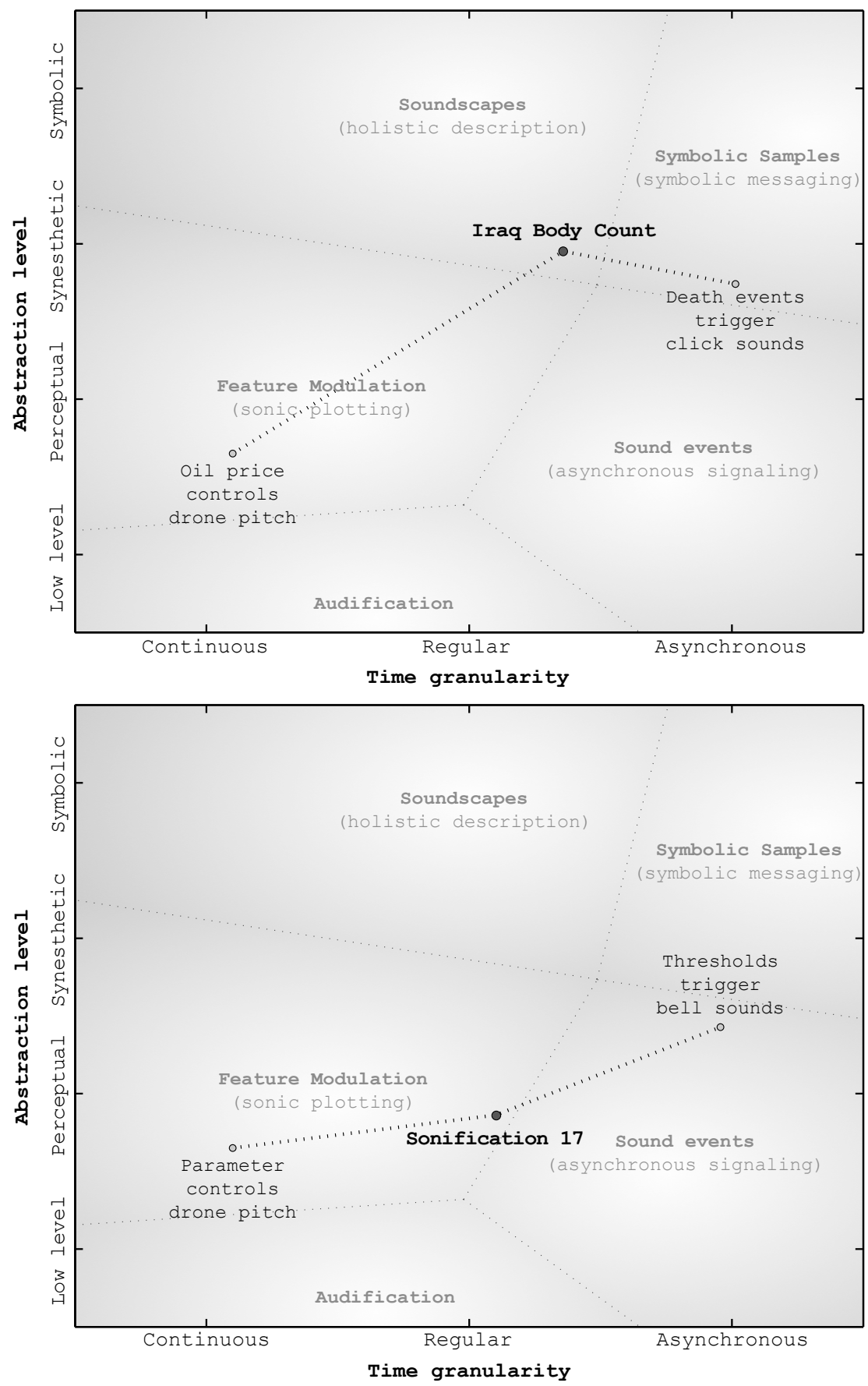

Figure 2: "Iraq Body Count" (top) and "Sonifying specific data points in a sequence along with the entire process" (bottom) in the sonification space. Light-gray circles represent sonification data bindings and the dark-gray ones indicates the main features. 


\subsection{Time Granularity}

The $x$ axis reflects the granularity of the events occurring in the sound and is measured in hertz. Sonifications where sound is likely to be a continuous modulation of features are located on the left part of the diagram, whereas the right part is devoted to discrete sound events in the sonification streams (generated by sparse data or interactive sonifications).

In order to verify the fitness of our proposal, we needed to measure the granularity of existing sonifications, borrowing the concept of TRAnsient Presence detection (TRAP), a metrics introduced in Music Information Retrieval and defined in [21]. This feature represents the amount of high-frequency content, calculated not on the signal itself but on its energy envelope. Such a measurement is performed by using CoBE [22], which can be interpreted as the amount of high frequencies in a signal.

Applying CoBE to the signal envelope, and not to the signal itself, reveals that continuous amplitude envelopes (where sound is likely to be a continuous modulation of features) have a low CoBE value, whereas crispy amplitude envelopes (corresponding to numerous sound-event onsets) present a high $\mathrm{CoBE}$ value. This concept is illustrated in Figure 3. From the CoBE value it is possible to infer the frequency of a sine wave having the same CoBE value of the input signal, namely the equivalent brightness frequency (EBF). The EBF-based representation makes such a measure independent from the signal sampling rate. For further details please refer to [21].

Finally, to place the main feature of a given sonification over the $x$ axis, we consider its average envelope EBF along time.

\subsection{Abstraction Level}

The $y$ axis relates to the level of abstraction. Real-world sounds are placed in the upper area, as their symbolic meaning is high (e.g. a dog bark), whereas synthesized sounds lay in the lower one, since they are typically far from our experience and we cannot bind them to a symbolical meaning (e.g. a frequency-modulated sine wave).

Since there is no commonly-accepted measurement unit for the symbolic content of a sound, we had to find out a heuristic to measure this parameter. We started from the assumption that the spectrogram of a real-world sound looks more complex than a synthesized one (see Figure 4). ${ }^{1}$ For this reason,

\footnotetext{
${ }^{1}$ The synthesized signal is a sine wave frequency-modulated by a high frequency signal,
} 


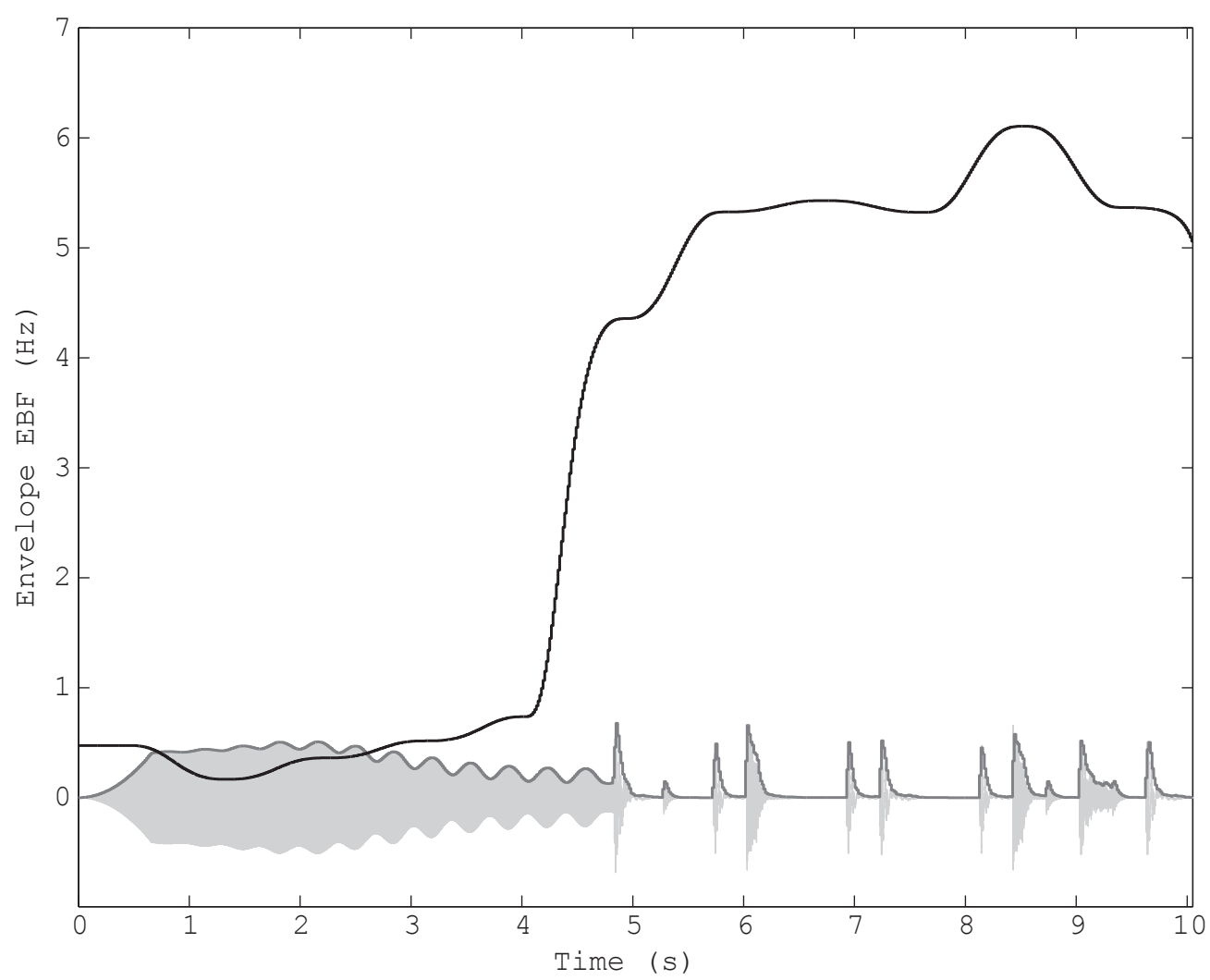

Figure 3: In gray the waveform and its amplitude envelope, in black the corresponding envelope EBF, interpretable as time granularity. The sound sample has been explicitly built in order to illustrate both low and high EBF values.

we measured the abstraction level of a sonification by calculating the entropy ${ }^{2}$ of its Short-Time Fast Fourier Transform interpreted as a gray-scale image.

Of course, some counterexamples can be found. For instance, comparing a bird tweet to a synthesized square wave, apparently the entire axis would be turned upside down: in fact the square wave is more spectrally complex than the natural bird sound. In order to solve this issue, we use the entropy of the spectrogram image instead of the signal's spectral entropy. In fact, the

\footnotetext{
thus presenting the typical harmonic content described by Bessel functions.

${ }^{2}$ Please note that this calculation of entropy is different from the one of spectral entropy commonly adopted in Music Information Retrieval tasks.
} 

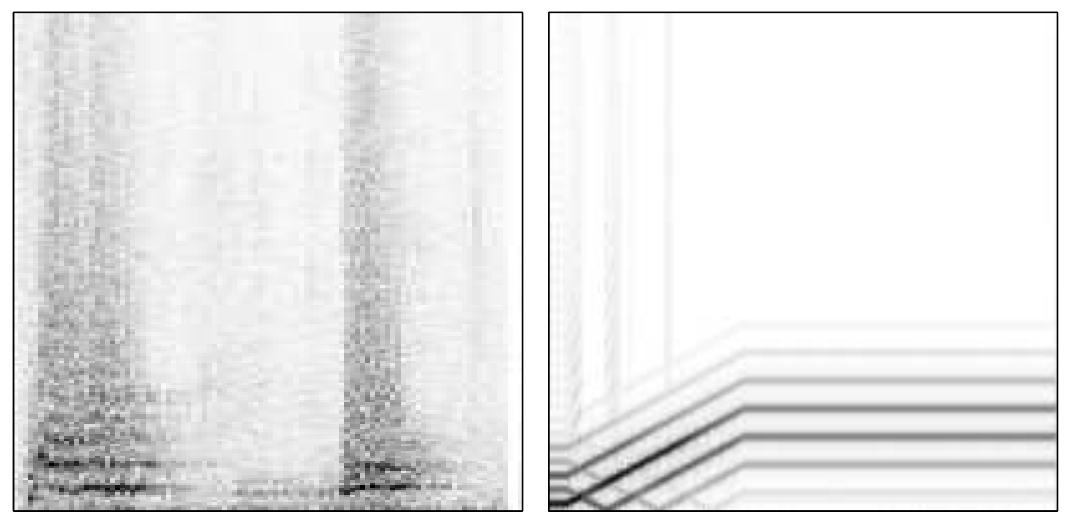

Figure 4: Spectrograms of a dog bark (left) and a frequency-modulated sine wave (right). The former diagram presents an entropy value higher than the latter one (1.9 and 0.7 respectively).

tweet produces a complex drawing while the square wave produces a regular pattern. However, some ambiguity may remain, since - as mentioned above - there is no signal feature directly related to the abstraction level.

\section{Validation of the Sonification Space}

In order to validate our approach from a qualitative point of view, the granularity and entropy characteristics from 22 sonification experiences mainly extracted from [20] - were analyzed automatically and placed in the reference system accordingly (see Figure 5). The aim of the validation is to compare the placement carried out automatically with that of subjective assessment of the original purpose of the sonification made by listening or interacting with them. A comparison between the two placements is then performed to identify weaknesses in the proposed model.

The list of the analyzed examples is shown in Table 1 . The average granularity is $6.993 \mathrm{~Hz}$ with a standard deviation of $2.751 \mathrm{~Hz}$, and the average entropy is 0.332 with a standard deviation of 0.205 .

The expected result was to highlight some link between sonic outcome (audio features) and sonification strategy. As a result, 17 examples out of $22(77.27 \%)$ have been placed according to their alleged original purposes, thus demonstrating the effectiveness of our approach. However, specific cases require some discussion. Sonification 05, 06, 10, 11, 21, and 22 are close to the Soudscapes area, and their placement is peculiar, but not wrong. In 


\begin{tabular}{|c|c|c|c|c|}
\hline ID & Name & TRAP & Entropy & Region \\
\hline 01 & $\begin{array}{l}\text { Sonification of Complex Biomedical } \\
\text { Data }\end{array}$ & 9.204 & 0.234 & $\mathrm{E}$ \\
\hline 02 & $\begin{array}{l}\text { Vocal Sonification of Pathologic EEG } \\
\text { Features }\end{array}$ & 5.767 & 0.217 & $\mathrm{~F}$ \\
\hline 03 & $\begin{array}{l}\text { C Programs Sonification using } \\
\text { Listen/C }\end{array}$ & 9.514 & 0.248 & $\mathrm{E}$ \\
\hline 04 & AuSOM for the Iris data set & 4.225 & 0.333 & $\mathrm{~F}$ \\
\hline 05 & $\begin{array}{l}\text { Principal Curve Sonification for the } \\
\text { Iris data set }\end{array}$ & 6.722 & 0.475 & $\mathrm{~S}, \mathrm{~F}$ \\
\hline 06 & Atmospherics/Weather Works & 4.519 & 0.782 & S \\
\hline$[07]$ & Geiger counter & 12.24 & 0.613 & $\mathrm{~F}$ \\
\hline 08 & The Shoogle mobile device & 8.816 & 0.446 & $\mathrm{Y}$ \\
\hline 09 & $\begin{array}{l}\text { Monitoring patients using auditory } \\
\text { icons }\end{array}$ & 5.041 & 0.190 & $\mathrm{~F}$ \\
\hline 10 & Weather Sonification of August 1940 & 4.838 & 0.653 & S \\
\hline 11 & Iraq Body Count & 9.433 & 0.482 & $\mathrm{~S}, \mathrm{~F}, \mathrm{Y}$ \\
\hline$[12]$ & $\begin{array}{l}\text { Sweatsonics Stream-Based } \\
\text { Sonification }\end{array}$ & 10.12 & 0.308 & $\mathrm{~F}, \mathrm{E}$ \\
\hline$[13]$ & Disconnection pulse probe & 6.044 & 0.135 & $\mathrm{Y}$ \\
\hline 14 & Auditory PCA Scatterplot & 10.01 & 0.208 & $\mathrm{E}$ \\
\hline 15 & Bat call & 6.626 & 0.011 & A \\
\hline 16 & Earthquake & 6.157 & 0.066 & $\mathrm{~A}$ \\
\hline$[17]$ & $\begin{array}{l}\text { Sonifying specific data points in a } \\
\text { sequence along with the entire process }\end{array}$ & 1.367 & 0.322 & $\mathrm{~F}, \mathrm{E}$ \\
\hline 18 & One-to-many mapping & 5.290 & 0.324 & $\mathrm{~F}$ \\
\hline [19] & Probing & 10.70 & 0.123 & $\mathrm{E}, \mathrm{F}$ \\
\hline 20 & Data Sonogram of the Iris data set & 8.148 & 0.112 & $\mathrm{E}$ \\
\hline 21 & $\begin{array}{l}\text { GNG sonification of the growth } \\
\text { process }\end{array}$ & 3.601 & 0.504 & $\mathrm{~S}, \mathrm{~F}$ \\
\hline 22 & stormyWinter & 3.549 & 0.528 & $\mathrm{~S}, \mathrm{~F}$ \\
\hline
\end{tabular}

Table 1: Sonification instances with Granularity and Abstraction measured by TRAP and Entropy respectively. The last column shows subjective positioning regions: A (Audification), E (sound Events), F (Feature modulation), S (Soundscapes), Y (sYmbolic samples). 


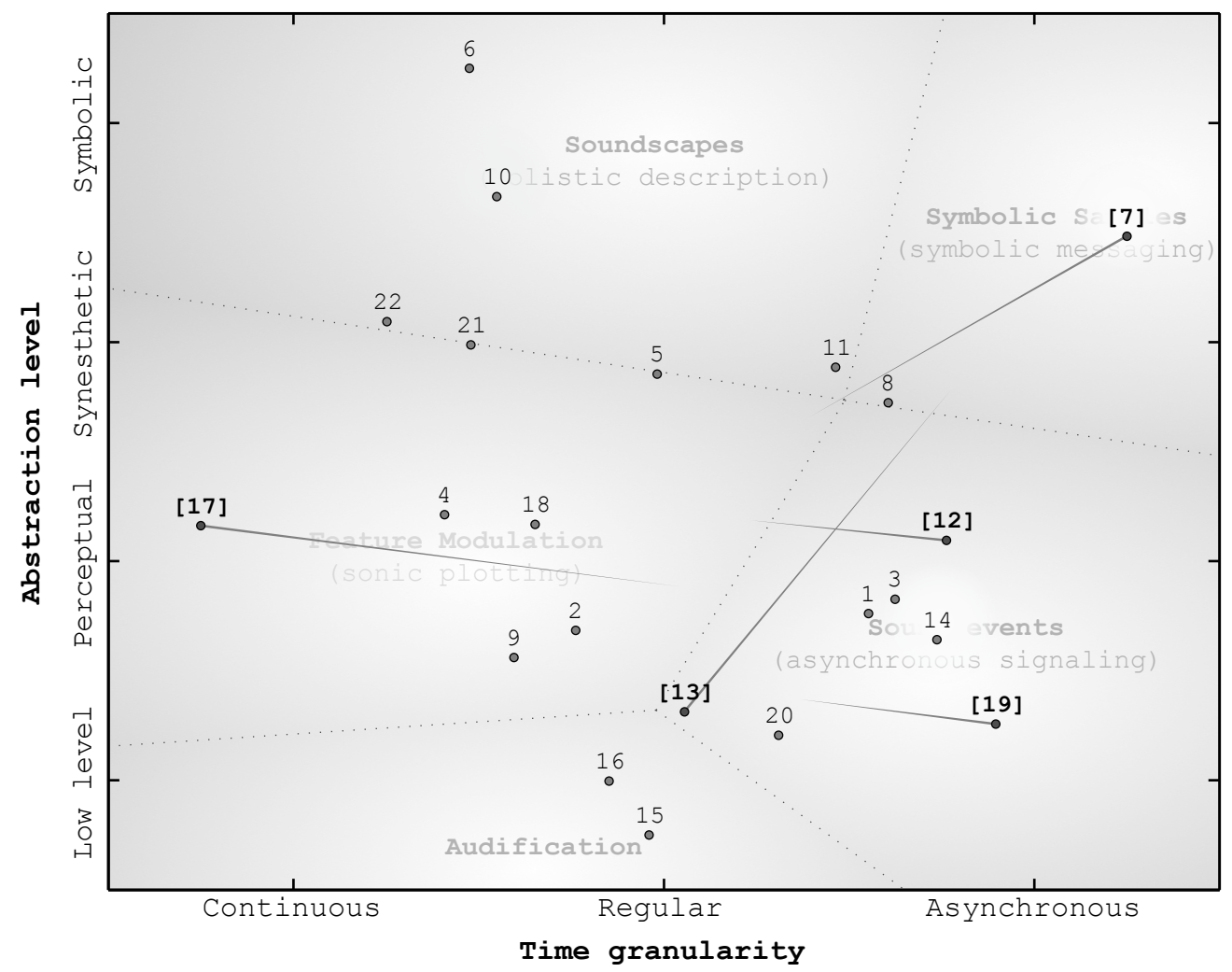

Figure 5: Automatic scattering of 22 sonification experiences over the sonification space. Parenthesized numbers identify misplaced sonification instances and needles point towards the right placement.

fact, they apply parameter-mapping and model-based techniques in order to build complex acoustical textures that mainly convey information about general system state. This case is well exemplified by Sonification 11, shown in Figure 2, where the combination of different techniques creates an artificial soundscape. Consequently, even if somehow borderline, the mentioned examples can be considered correctly placed. Moreover, Sonification 08 has been placed near the correct class, but slightly biased towards the center, since it consists of a soundbank of sounds stored very close to each other and sorted by type; in a real-world context they would be more scattered and heterogeneous, and the example would have a better placement.

Mapping relevant case studies over the proposed sonification space can 
also be a way to unveil interesting aspects of sonification. For instance, Sonification 15 describes a bat performing a systematic scan of the surroundings. Originally, pitch and delay time are modulated by the bat and the surrounding environment depending on speed and distance parameters: this is a typical sonification strategy. On the other hand this sound can be heard by human beings only after a proper pitch shift, which is an audification task. Consequently this example shares the characteristics of both sonification and audification domains.

For the 5 misplaced examples (with bold parenthesized labels in Figure 5), the nature of the source data and the sounds used to represent them make automatic classification hard. Sonification 12 and 19 seem to be discrete parameter mapping whose value of time granularity has been overestimated. Sonification 13, essentially an alarm, could be either an earcon or an auditory icon, but the characteristics of the produced sound - a simple square wave push it towards the lower part of the diagram.

Sonification 17's granularity was tracked incorrectly: the algorithm apparently ignored the asynchronous events occurring within this instance. The algorithm should be refined to take into account also the discontinuities of other features.

Finally, Sonification 07 - namely the Geiger counter sonifying the detection of alpha and beta particles - presents a clear discrepancy between the synthesis technique and the perceived sound results. Regarding the former aspect, the example is correctly located in the sonification space, since the Geiger counter emits a click sound for each detected particle, thus behaving like an auditory icon. On the contrary, the information we gather from the sound is based on the time granularity of detection events, like parametermapping sonifications. In this case, a way to represent the Geiger counter in the sonification space would be to put the data binding in the Sound events area and the main feature in the Feature modulation area.

\section{Conclusions and Future Work}

In this work we described a reference system useful both to interpret already-existing sonification instances and to plan new sonification tasks. To this goal, we introduced a conceptual framework called the sonification space. Two dimensions have been considered to locate entities, namely the level of abstraction and the time granularity. 
The resulting model can be used to classify sonification techniques and their instances, to support meta-studies on sonification (e.g. to assess how successful particular techniques have been), to sketch new sonification strategies, and to document specific experiences. In particular, a computer-aided sonification system should help sonification activities either by analyzing the correlation among data and proposing soundscapes that intrinsically present features with similar correlation, or by proposing efficient strategies in analogous scenarios. Another application is the automatic suggestion of which features to map in order to be clearly distinguishable.

The validation conducted on a number of available examples showed encouraging results, however a more formal and detailed activity is required in order to achieve the aforementioned goals. We are planning to improve the proposed space in order to foster the creation of layered sonifications and to implement an effective recommender system for sonification.

Moreover, a number of open issues affects different areas of the sonification space. As it regards feature modulation (sonic plotting), it is worth citing:

1. Orthogonality [13] - Which sound parameters are orthogonal, if any? The orthogonality problem can be addressed by studying which acoustic features are independent, given the sonification of an uncorrelated dataset. $^{3}$

2. Perceptibility [6] - How many different sound features can be perceived by a common listener, and how precisely? What is the maximum number of polyphonic voices that can be exploited to convey multichannel information? Our approach will be testing if users can track single and multiple sound-feature trajectories. In the field of psychoacoustics, many researches have been conducted to address this issue (see e.g. [23], [24], and [25]), and their findings should be highly regarded in sonification activities.

3. Evolutionary bindings [16] - Which sound features are the most adequate to represent physical quantities and phenomena? Chosen features should exploit our evolutionary and cultural endowment in order to improve comprehension even by untrained listeners, as stated in [10]. Moreover, mapping the same data to more than one feature may re-

\footnotetext{
${ }^{3}$ In the scientific community, the possibility to find out a set of independent sound parameters is a matter of debate.
} 
inforce the message and reduce ambiguity by creating a more realistic scenario [3]. This study is necessary to improve sonification learning curve and to enable affective interaction.

As it regards soundscaping (system state description) and symbolic samples, open issues are:

1. Lack of guidelines and metaphors - Not all data to be sonified have an effective counterpart in the physical properties that can be inferred from sound. There are many datasets (like bitmaps, quantum physics and chemical data) which must relay on other cues. In some cases music language can provide a useful tool [26]. In fact there are brain regions - responsible for the mechanism of expectations - that can learn harmonic patterns while listening to a coherent music fragment, and generate tension potential when music violates its internal coherence $[16,19]$. Another solution to this problem is the adoption of natural systems (soundscapes) that show features similar to the dataset to sonify.

2. Cultural boundaries [27] - Different natural soundscapes train different hearing skills, consequently using soundscapes as metaphors in sonification may arise localization problems.

Future work will focus on the mentioned open issues, in order to transform this theoretical work into an effective computer-based recommender system for sonification.

[1] G. Kramer, B. Walker, T. Bonebright, P. Cook, J. Flowers, N. Miner, J. Neuhoff, R. Bargar, S. Barrass, J. Berger, et al., The sonification report: Status of the field and research agenda. report prepared for the national science foundation by members of the international community for auditory display, International Community for Auditory Display (ICAD), Santa Fe, NM.

[2] T. Hermann, Taxonomy and definitions for sonification and auditory display, Proceedings of the 14th International Conference on Auditory Display (ICAD 2008), IRCAM, 2008.

[3] G. Kramer, Mapping a single data stream to multiple auditory variables: A subjective approach to creating a compelling design, in: International Conference on Auditory Displays, Palo Alto, California, USA, 1996. 
[4] T. Madhyastha, D. Reed, A framework for sonication design, Auditory Display: Sonification, Audification and Auditory Interfaces (1994) 267290.

[5] S. Barrass, Sculpting a sound space with information properties, Organised sound 1 (02) (1996) 125-136.

[6] J. G. Neuhoff, G. Kramer, J. Wayand, Sonification and the interaction of perceptual dimensions: Can the data get lost in the map, in: International Conference on Auditory Display, 2000.

[7] G. Kramer, Some organizing principles for auditory display, Auditory Display: Sonification, Audification, and Auditory Interface. SFI Studies in the Sciences of Complexity, Proc. XVIII (1994) 185-221.

[8] S. Smith, Representing data with sound, in: Proceedings of IEEE Visualization, IEEE CS Press, Piscataway, N.J., 1990.

[9] G. S. Kendall, Visualization by ear: Auditory imagery for scientific visualization and virtual reality, Computer Music Journal (1991) 70-73.

[10] T. Hermann, H. Ritter, Listen to your data: Model-based sonification for data analysis, Advances in intelligent computing and multimedia systems 8 (1999) 189-194.

[11] A. Dewitt, R. Bresin, Sound design for affective interaction, in: Affective Computing and Intelligent Interaction, Springer, 2007, pp. 523-533.

[12] N. Degara, T. Kuppanda, T. Neate, J. Yang, A. V. Torres, Reproducible sonification for virtual navigation, in: VR Workshop: Sonic Interaction in Virtual Environments (SIVE), 2014 IEEE, IEEE, 2014, pp. 35-40.

[13] G. Kramer, Auditory Display: Sonification, Audification, and Auditory Interface. SFI Studies in the Sciences of Complexity, Proc. XVIII, Addison-Wesley, 1994.

[14] G. E. Blattner MM, Papp AL, Sonic enhancement of two-dimensional graphic display, Auditory Display: Sonification, Audification, and Auditory Interface. SFI Studies in the Sciences of Complexity, Proc. XVIII (1994) 447-470. 
[15] D. R. Bach, H. Schächinger, J. G. Neuhoff, F. Esposito, F. Di Salle, C. Lehmann, M. Herdener, K. Scheffler, E. Seifritz, Rising sound intensity: an intrinsic warning cue activating the amygdala, Cerebral Cortex 18 (1) (2008) 145-150.

[16] P. N. Juslin, D. Västfjäll, Emotional responses to music: The need to consider underlying mechanisms, Behavioral and brain sciences 31 (05) (2008) 559-575.

[17] D. W. Ploog, The evolution of vocal communication, Nonverbal vocal communication: Comparative and developmental approaches (1992) 6 .

[18] A. R. Damasio, B. Everitt, D. Bishop, The somatic marker hypothesis and the possible functions of the prefrontal cortex [and discussion], Philosophical Transactions of the Royal Society of London. Series B: Biological Sciences 351 (1346) (1996) 1413-1420.

[19] P. Janata, J. L. Birk, J. D. Van Horn, M. Leman, B. Tillmann, J. J. Bharucha, The cortical topography of tonal structures underlying western music, Science 298 (5601) (2002) 2167-2170.

[20] T. Hermann, A. Hunt, J. G. Neuhoff, The sonification handbook, Logos Verlag Berlin, 2011.

[21] G. Presti, D. Mauro, G. Haus, TRAP: TRAnsient Presence detection exploiting Continuous Brightness Estimation (CoBE), in: Proceedings of the 12th Sound and Music Computing Conference (SMC 2015), Maynooth, Ireland, 2015.

[22] G. Presti, D. Mauro, Continuous Brightness Estimation (CoBE): Implementation and its possible applications, in: 10th International Symposium on Computer Music Multidisciplinary Research (CMMR), Laboratoire de Mécanique et d'Acoustique, 2013, pp. 967-974.

[23] A. S. Bregman, Auditory scene analysis: The perceptual organization of sound, MIT press, 1994.

[24] J. H. McDermott, A. J. Oxenham, Music perception, pitch, and the auditory system, Current opinion in neurobiology 18 (4) (2008) 452463. 
[25] D. Wang, G. J. Brown, Computational auditory scene analysis: Principles, algorithms, and applications, Wiley-IEEE Press, 2006.

[26] R. Nikolaidis, B. Walker, G. Weinberg, Generative musical tension modeling and its application to dynamic sonification, Computer Music Journal 36 (1) (2012) 55-64.

[27] R. M. Schafer, The soundscape: Our sonic environment and the tuning of the world, Inner Traditions/Bear \& Co, 1993.

\section{Appendix}

In this section we acknowledge the authors of the sonification examples listed in Table 1 and partially extracted from [20].

1. Sonification of Complex Biomedical Data by Gerold Baier, Thomas Hermann and Ulrich Stephani

2. Vocal Sonification of Pathologic EEG Features by Thomas Hermann, Gerold Baier, Ulrich Stephani and Helge Ritter

3. C Programs sonified using Listen/C by David B. Boardman, Geoffrey Greene, Vivek Khandelwal and Aditya P. Mathur

4. AuSOM for the Iris data set by Thomas Hermann, Thomas Henning and Helge Ritter

5. Principal Curve Sonification for the Iris data set by Thomas Hermann, Peter Meinicke and Helge Ritter

6. Atmospherics/Weather Works by Andrea Polli, Glenn Van Knowe and Chuck Varga

7. Geiger counter (public sample)

8. The Shoogle mobile device by Rod Murray-Smith and John Williamson

9. Monitoring patients using auditory icons: Sonifying the Body Electric by Gregory Kramer

10. Weather sonification of August 1940 by John Flowers

11. Iraq Body Count by Guillaume Potard

12. Sweatsonics Stream-Based-Sonification

13. Disconnection pulse probe recorded by Claude Blancard for Anne Guillaume 
14. Auditory PCA Scatterplot by Sam Ferguson, William Martens and Densil Cabrera

15. Bat call by dobroide@freesound.org

16. Earthquake from Sonifyer.org

17. Sonifying specific data points in a sequence along with the entire process by Jonathan Berger

18. One-to-many mapping by F. Grond, T. Bovermann and T. Hermann

19. Probing by R. J. Cassidy, J. Berger, K. Lee, M. Maggioni and R. R. Coifman

20. Data Sonogram of the Iris data set by Thomas Hermann

21. GNG Sonification of the growth process by Thomas Hermann

22. stormyWinter by Thomas Hermann, Jan M. Drees and Helge Ritter 